Escuela de Ciencias Agrícolas, Pecuarias y del Medio Ambiente
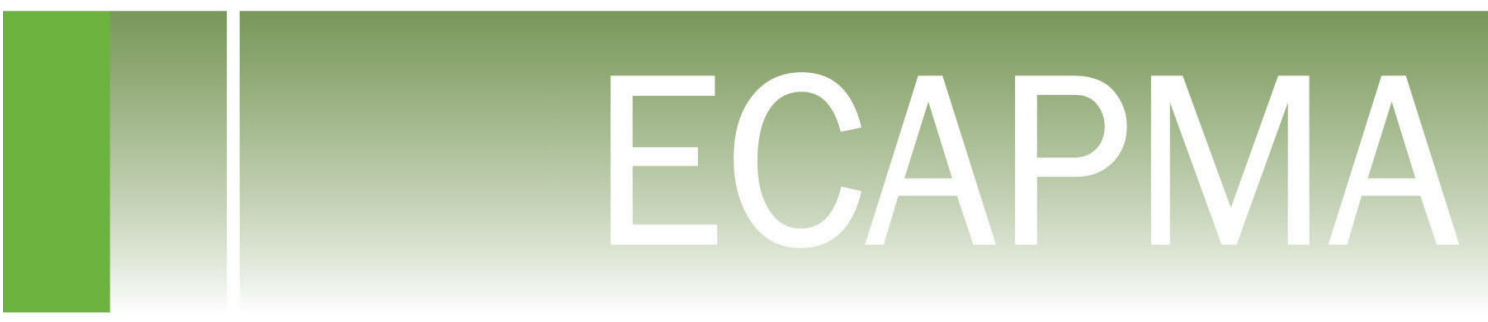



\title{
APLICACIÓN DE BUENAS PRÁCTICAS AGRÍCOLAS EN CEBOLLA CABEZONA (Allium cepa) Y TOMATE DE MESA (Lycopersicum esculentum)
}

\author{
Gloria María Cifuentes ${ }^{88}$ y Molano José Herrán ${ }^{89}$
}

\begin{abstract}
RESUMEN
En el marco del proyecto Observatorio de Cadenas Productivas hortofrutícolas se ha querido contribuir con el análisis de las Buenas Prácticas Agrícolas en los cultivos de cebolla cabezona y de tomate de mesa tanto en condiciones a campo abierto como en invernadero. Las Buenas Prácticas Agrícolas BPA corresponden a las actividades que pretenden mejorar la producción en cada una de sus diferentes procesos de manera que se reduzca el riego de toxicidad en los productos y así generar productos inocuos en la salud. Este escrito presenta la descripción de las problemáticas que se presentan en cuanto a la aplicación de las BPA en los dos cultivos seleccionados, buscando encontrar respuestas a la manera como el agricultor maneja los diferentes procesos. Se presenta entonces un resumen de los principales resultados que tienen relación con preguntas de investigación cómo:
\end{abstract}

¿En qué consiste la aplicación de las BPA?, ¿cuál es el impacto de las BPA en el suelo, agua, aire, recursos naturales?, ¿cuál es la incidencia de las BPA en la calidad de la Cebolla cabezona Alliun cepa y en el tomate de mesa Lycopersicum esculentum?, ¿cómo inciden las BPA en la salud de los productores y de sus familias?

El trabajo se realiza en la región de Alto Ricaurte y Valle de Suamux en donde se evidencia la problemática de los productores en los diferentes eslabones de la cadena referida a aspectos como el control y ajuste de la humedad y cantidad de luz en sistemas de invernadero, para el primero se requiere de 65 a $80 \%$ de humedad, y días con un buen porcentaje de luminosidad para favorecer la fotosíntesis, en caso contrario la planta disminuye procesos de floración y absorción de nutrientes. La ventilación es otro aspecto a tener en cuenta dependiendo del área y del microclima existente e incide en la floración e incidencia de plagas y enfermedades.

Es fundamental poner especial atención a la flora circundante que hace parte de los agrosistemas pues son ecosistemas encargados de proteger los cultivos como barreras vivas, albergar insectos benéficos y proteger los almacenamientos de agua.

La cebolla de bulbo prospera en suelos fértiles, con buen drenaje y con textura franca arcillosa. Se hace necesario su especial cuidado ya que subsisten procesos erosivos bastante graves que presentan erosión eólica, erosión por laboreo irracional del suelo y falta de manejo para desarrollar sistemas agrícolas y ecológicos sustentables.

88 Ingeniera Agrónoma. Universidad Nacional Abierta y a Distancia. Gloria.cifuentes @unad.edu.co. Grupo de investigación GICAFAT. COL0023054. Línea de Investigación Cadenas productivas. ECAPMA

89 Escuela de Ciencias Agrícolas, Pecuarias y del Medio Ambiente, ECAPMA, UNAD. 
Es conveniente fortalecer las estructuras asociativas en aras de mejorar las relaciones comerciales, como la intermediación, obtener buenos precios y servicios para los productores, igualmente el bienestar, la salud de los trabajadores y sus familias es fundamental y se hace indispensable realizar un análisis de este factor que es transversal dentro de los factores de BPA que influyen en la producción agrícola.

Palabras clave: Buenas Prácticas Agrícolas, Allium cepa, Lycopersicum esculentum, manejo de plagas, enfermedades, prácticas culturales, suelo, cosecha, poscosecha, comercialización, salud.

\begin{abstract}
In the frame of the project Observatory of Productive "hortifrutiolas" Chains was wanted to be contributed by the analysis of the Good Agricultural Practices in the cultures of onion and of tomato not only in conditions to field opened but also in greenhouse. The Good Agricultural Practices GAP corresponds to activities that aim to improve the production in each of its various processes so as to reduce the risk of toxicity in products and generate health-friendly products. This paper presents a description of the problems that arise in the application of GAP in the two selected crops, seeking answers to how the farmer manages the different processes.
\end{abstract}

So it is present a summary of the main results that relate to research questions like: What is the application of GAP? What is the impact of GAP in soil, water, air, natural resources? What is the impact of GAP on the quality of onion (Alliun strain) and table tomatoes (Lycopersicon esculentum)? How GAP impact on the health of farmers and their families?

The work is done in the region of Alto Ricaurte and Suamux Valley where evidence of the problems of producers in the various links in the chain reified aspects such as control and adjust the humidity and amount of light in greenhouse systems, required for the first 65 to $80 \%$ humidity, and days with a good percentage of light to support photosynthesis, otherwise the process of flowering plant and decreases absorption of nutrients. Ventilation is another aspect to consider depending on the area and the existing microclimate and influences flowering and incidence of pests and diseases.

It is essential to pay special attention to the surrounding flora that is part of the agroecosystems as they are assigned to protect crops such as barriers, host beneficial insects and protect the storage of water.

The onion bulb thrives on fertile, well drained and loamy clay, it is necessary that his particular care and remain quite severe erosion that present wind erosion, tillage erosion irrational and lack of land to develop agricultural systems management and ecologically sustainable. It is necessary to strengthen partnership structures environment to improve trade relations, such as brokering, get good prices and services to producers, also the welfare, health workers and 
their families is essential and indispensable to analyze this factor that is transverse within GAP factors that influence agricultural production.

Key words: Good Agricultural Practices, Allium cepa, Lycopersicum esculentum, pest management, diseases, cultural practices, soil, harvest, post harvest, marketing, health.

\section{INTRODUCCIÓN}

La cebolla cabezona Allium cepa es un cultivo de hortaliza explotado en varios municipios de Boyacá entre ellos Sáchica, Sutamarchán, Tinjacá, Villa de Leyva, Valle de Sogamoso, Duitama, Toca, Tuta, Sotaquirá, Ventaquemada y Pesca, entre otros municipios. El Tomate Lycopersicum esculentum $\mathrm{L}$ por su parte, está siendo cultivado en invernaderos y a cielo abierto. El tomate larga vida tiene amplia cobertura en municipios como Santa Sofía, Sáchica, Sutamarchán, Villa de Leyva, Valle de Tenza, Garagoa, La Capilla, Chinavita y Provincia del Norte de Boyacá: San Mateo, Boavita, Guacamayas, entre otros. Se ha detectado, dentro de la problemática, daño en el sustrato sobre el cual se cultiva, afectación en la micro-flora de suelo, por mal manejo de los insumos agrícolas como los fertilizantes, aplicación de sustancias para la desinfección del mismo que ocasionan resistencia a patógenos. Por otra parte, la aplicación de agua de riego ha sido implementado sin tener en cuenta la racionalización del recurso, causando graves daños al suelo como lixiviación de nutrientes y encharcamientos.

El estudio se justificó dada la importancia de los volúmenes de producción, la cantidad de mano de obra empleada en las diferentes labores y el uso intensivo del suelo en predios que oscilan entre los 0.5 y 2 Ha en su gran mayoría. Es necesario dar una mirada a la aplicación de las BPA que intentan identificar en qué medida se están aplicando y cómo contribuyen a mejorar la producción y los niveles de vida del agricultor. Existen algunos estudios que sobre BPA se han realizado, tales como: análisis sobre uso de material en donde se utilizaron 5 híbridos de tomate larga vida en Palmerillas en el municipio de Eljido, Almería en España, ensayo realizado bajo invernadero recubierto con malla y polietileno, el experimento permite encontrar índices de Carbonato de calcio residual de $-0.16 \mathrm{meq} / 1$, dureza del agua de 17,62 rango con un coeficiente de alcalinometría de 10 que se considera de buena calidad. Otro de los ensayos realizados y que contribuyen a las BPA es el estudio sobre aplicaciones de magnesio y micronutrientes $\mathrm{B}, \mathrm{Zn}, \mathrm{Mn}$ en programas de fertilización en cebolla cabezona con el fin de mejorar la calidad en el producto.

Como hipótesis se plantea que la identificación del grado de adopción de las BPA en los cultivos Allium cepa y Lycopersicum esculentum favorecen la implementación de acciones en torno al mejoramiento de los diferentes procesos involucrados en la producción y comercialización de los productos.

La aplicación de las BPA reviste gran interés para las asociaciones de productores en razón a que favorece la calidad del producto, y son básicas en los mercados sobre todo en los de carácter internacional, para proporcionar productos inocuos, sanos, derivados de un trabajo consiente 
por parte de las comunidades productoras que unidos sus esfuerzos a las políticas de líderes gubernamentales y educativos procuren una producción ambientalmente sana y con mayor bienestar para los trabajadores. El trabajo está inmerso en la línea de investigación Cadenas productivas y realizado dentro del grupo GICAFAT.

\section{OBJETIVO GENERAL}

Realizar un diagnóstico de la situación actual en el manejo de las Buenas Prácticas Agrícolas BPA, en los cultivos de cebolla cabezona Allium cepa y tomate de mesa Lycopersicum esculentum $L$.

\section{OBJETIVOS ESPECÍFICOS}

Reconocer la importancia de las BPA en los cultivos cebolla cabezona Allium cepa y tomate de mesa Lycopersicum esculentum $L$.

Establecer que la brecha tecnológica respecto a la aplicación de las BPA en los cultivos de cebolla cabezona (Allium cepa L) y tomate de mesa Lycopersicum esculentum puede impedir el mejoramiento de estos cultivos.

\section{MATERIALES Y MÉTODOS}

Tipo de estudio: corresponde al inductivo -deductivo; para tal fin se realizaron visitas a los predios para tomar anotaciones en registros y se adelantaron conversaciones con los productores de Sáchica, Santa Sofía, y El Valle de Sogamoso con el fin de analizar las buenas prácticas que se aplican en preparación de suelo, preparación de semilleros, trasplante, fertilización, lo mismo que en el uso de plaguicidas, manejo de plagas y enfermedades y cosecha. Se observaron igualmente las prácticas de pos cosecha, empaque y embalaje del producto y se aplicaron encuestas que identificaban aspectos relacionados con los procesos de producción, cosecha, almacenamiento, uso de agroquímicos, manejo de los recursos naturales y comercialización entre otros.

Método de investigación: de carácter descriptivo. A través de la observación se logró la identificación de aspectos que inciden en el manejo de procesos y procedimientos que involucran mano de obra, uso de recursos naturales y mercadeo, entre otros.

Análisis estadístico: se tabularon las encuestas para somete a análisis al conjunto de datos recolectados en campo. Se realizaron observaciones aleatorias y muestreo para poder inferir estadísticamente algunas situaciones presentadas con la aplicación de las BPA en los cultivos objeto de la investigación con medidas de tendencia central, dispersión y algunas medidas de posición. 


\section{RESULTADOS Y DISCUSIÓN}

El tomate larga vida se cultiva ampliamente en Boyacá;, de la zona de Alto Ricaurte se distribuye en Sutamarchán, Sáchica, y especialmente Santa Sofía y Villa de Leyva y representa el 15,84 del área sembrada en hortalizas. Los sistemas de producción bajo invernadero son característicos de la zona y generan un alto volumen de mano de obra con alrededor de 155 jornales por Ha y ciclo de producción. El rendimiento se estima en $5 \mathrm{Kg}$ de tomate por planta incrementándose la producción bajo invernadero según el microclima creado y el manejo al cultivo.

\section{Análisis de las condiciones agroecológicas}

El $80 \%$ de los agricultores de tomate tienen en cuenta las condiciones de temperatura imperantes en la región, sin embargo, no la consideran factor preponderante por cuanto la temperatura adecuada la logran con condiciones bajo cubierta. No existen técnicas adecuadas para el manejo de temperatura versus humedad mediante mecanismos de ventilación técnicamente asistidos, se manejan estas variables a priori, y con medidas eventuales de rangos de temperatura y humedad aportada por el riego. La precipitación es un factor importante en el cultivo de la cebolla especialmente en el valle de Suamux, pero el agricultor cuenta con sistemas de riego para el caso de la cebolla cabezona Allium Cepa. En Sáchica y Sutamarchán los cultivadores de cebolla dependen especialmente de reservorios construidos para almacenar agua en épocas críticas ya que en esta zona debido al fenómeno de Sotavento la lluvia es escaza, alrededor de $500 \mathrm{~mm}$ por año.

La humedad relativa debe permanecer en los cultivos de invernadero entre 60 y $80 \%$ y se regula con ventanas o con zonas semi-cubiertas que regulan la temperatura. Un $30 \%$ de los agricultores toma en cuenta esta variable para ajustar los valores exigidos en tomate bajo invernadero. Igualmente el $20 \%$ de los agricultores toman en cuenta las condiciones de temperatura y brillo solar y el 10\% solamente pone atención a los aspectos relacionados con la ventilación, ya que no cuentan con sistemas para adecuar estas variables de manera que el cultivo de tomate pueda adaptarse a las mejores condiciones agroclimáticas.

\section{Consideraciones sobre ubicación del cultivo e infraestructura}

Los agricultores delimitan el área y hacen empíricamente un análisis de la conveniencia del lugar para establecer el invernadero. Sin embargo, se orientan por las decisiones tomadas por el agricultor vecino y por agricultores exitosos, por lo tanto, es necesario capacitarlos para que pueda identificar técnicamente el área en donde se instalará el cultivo. Normalmente en un $85 \%$ de casos los agricultores no hacen rotación de cultivos, labor tan fundamental para evitar la proliferación y permanencia de los patógenos en el suelo y el desgaste de los sustratos edáficos en cuanto a nutrientes. (Ver Figura 1).

El 90 \% no cuenta con un mapa estructurado de la finca en donde se detallen las zonas de especial manejo, como bosques, fuentes de agua, y zonificación de suelos según su uso e 
historia de los suelos y del cultivo. El 10\% de los agricultores no practica el análisis riguroso de suelo que les indica la cantidad de nutrientes que tiene el suelo para cultivo de cebolla cabezona y tomate o el sustrato utilizado en los invernaderos.

Figura 1. Baja rotación de suelos en cultivos de cebolla.

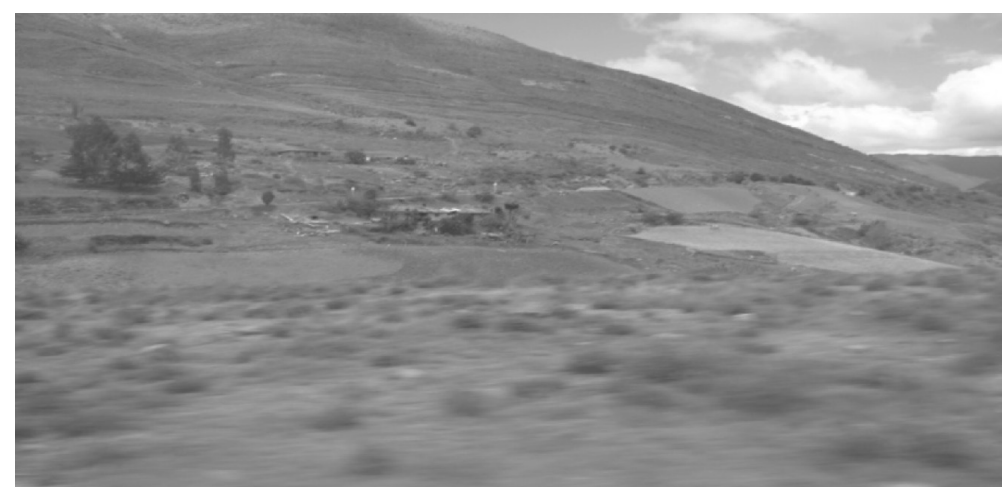

E1 90\% de los agricultores tampoco practica análisis físico y sólo el 5\% realiza un análisis microbiológico de las aguas para riego. Algunos como los cultivadores de tomate larga vida toman el agua de riego de acueductos veredales o municipales, en tanto que los productores de cebolla cabezona la toman de sendos reservorios construidos en zonas arcillosas.

Las fincas cuentan con instalaciones de sanitario, en un 70\%, un $80 \%$ no cuenta con lavamanos y en su defecto se encuentran infraestructuras como lavaderos que cumplen el mismo fin. En un $20 \%$ de fincas existen las duchas para los trabajadores, el $80 \%$ no cuenta con este servicio, sólo existe el de las familias. Los implementos de protección para los trabajadores se evidencian en un $30 \%$ en los cultivos de precisión, y escasamente en los cultivos a campo abierto en donde utilizan su propia ropa o vestimenta para realizar las labores.

Las fincas cuentan con cuartos en un $70 \%$ en donde se almacena la herramienta y los equipos como motobombas, allí mismo se almacenan fertilizantes e insumos como plaguicidas y fungicidas en forma desordenada y acarreando riesgos para los niños, especialmente.

El $25 \%$ de las fincas productoras cuenta con sitios adecuados para almacenar el tomate y la cebolla cabezona, entre $80 \%$ y $90 \%$ no cuenta con instalaciones adecuadas con temperatura, índice de humedad, luz, adecuado para almacenar sus productos.

En las fincas se observan las canastillas, costales y empaques para la recolección de los productos, pero en un $80 \%$ de estas no se realiza la desinfección de las canastillas y del empaque utilizado. El 90\% no cuenta con higrómetro, pluviómetro ni trampas para insectos y roedores. 
El 90\% de las fincas productoras de cebolla cabezona y tomate no recopilan sistemáticamente la información ni la registran en formatos de valoración y evaluación de riesgos para valorar las situaciones relacionadas con la planeación de la finca.

\section{BPA en el manejo del cultivo}

La densidad de siembra para tomate larga vida es la adecuada en un $90 \%$ de las fincas productoras de tomate bajo invernadero, igualmente en cebolla cabezona se manejan densidades de siembra adecuadas. En el manejo del cultivo se tienen en cuenta algunos aspectos como el uso de insumos para fertilización, plaguicidas, insecticidas y fungicidas que son aplicados al cultivo para el control de algunos patógenos que ocasionan daño al cultivo.

\section{Análisis de BPA en preparación del suelo}

La biodiversidad se ha visto en peligro debido a la ampliación de la frontera agrícola y al hecho de no considerar el cuidado y preservación de los recursos que están de alguna manera asociados con los sistemas productivos, pues de ellos depende, en gran parte, el equilibrio ecológico, el albergue de insectos, hongos y bacterias benéficas, lo mismo que la flora silvestre.

En lo referido a registro de labores de cultivo se puede verificar que sólo un $10 \%$ de los cultivadores de tomate larga vida llevan datos relacionados con la fecha de realización de labores culturales, el motivo, los equipos utilizados y los formatos correspondientes a la aplicación fitosanitaria y de abonos foliares y edáficos. Es necesario evidenciar en los registros la fecha de aplicación, el nombre del producto, el ingrediente activo que contiene el producto, lo mismo que el motivo por el cual se aplica la dosis en términos de gramos por planta y el total, la mezcla de agua, la presión utilizada en los equipos de bombeo, y el nombre del operario que hace la aplicación.

La implementación de la BPA se realiza para asegurar la competitividad de los productos en aras de mejorar los mercados nacionales y para exportar bajo condiciones de inocuidad. Existen puntos de control como:

\section{Uso de fertilizantes orgánicos}

El uso de abonos orgánicos si bien mejora la estructura de los suelos, proporciona elementos mayores y menores a la planta y dinamiza la vida de los microorganismos en el suelo, mejorando las interrelaciones entre estos y los minerales, ocasiona transmisión de algunas bacterias, hongos y nematodos nocivos para la salud, en lo posible debe sufrir un proceso de compostación antes de aplicarla al suelo para que se potencien los organismos benéficos y se puedan pasterizar los sustratos para disminuir la incidencia de patógenos. En el cultivo de cebolla de bulbo se aplica abono orgánico especialmente gallinaza con un bajo nivel de 
descomposición, y no se realiza evaluación de riesgos para tomar en consideración su origen, tampoco se analizan sus características.

\section{El uso de fertilizantes}

Este se fundamenta en la disponibilidad de nutrientes en el suelo y en los requerimientos de la planta, para ello el análisis de suelo es importante. En las fincas de cultivo de tomate en un $15 \%$ se lleva el registro de uso fertilizantes edáficos y fertilizantes foliares, en cebolla los agricultores no llevan registros en un gran mayoría, sólo un 5\% lo hace en la unidad productiva. Se observa que los fertilizantes son almacenados en sitios protegidos pero no están separados de los plaguicidas, fungicidas, herbicidas, y demás productos químicos y biológicos que se utilizan en la finca. Tampoco se evidencia letreros o avisos que informen de manera clara la identificación por grupos de productos ni indicaciones respecto al cuidado para la salud y al uso mismo de los productos.

\section{Aplicación de riego}

En tomate larga vida, en el $60 \%$ de las explotaciones, mantiene niveles apropiados de humedad en la planta a través de proporcionar fertirrigación de modo que se manejan láminas de riego adecuadas. En cebolla de bulbo el 85\% de los productores no lleva a cabo mediciones periódicas para calcular las necesidades de agua al cultivo. Tampoco se tiene en cuenta la humedad del suelo ni las predicciones de precipitación en el área de cultivo. No se conoce el grado de evaporación del cultivo ni del suelo para calcular necesidades de riego. El uso del agua en ocasiones es irracional y se causan desperdicios del recurso, encharcamiento de los suelos con los consecuentes daños especialmente al suelo. (Figura 2).

Figura 2. Procesos de encharcamiento de los surcos en tomate

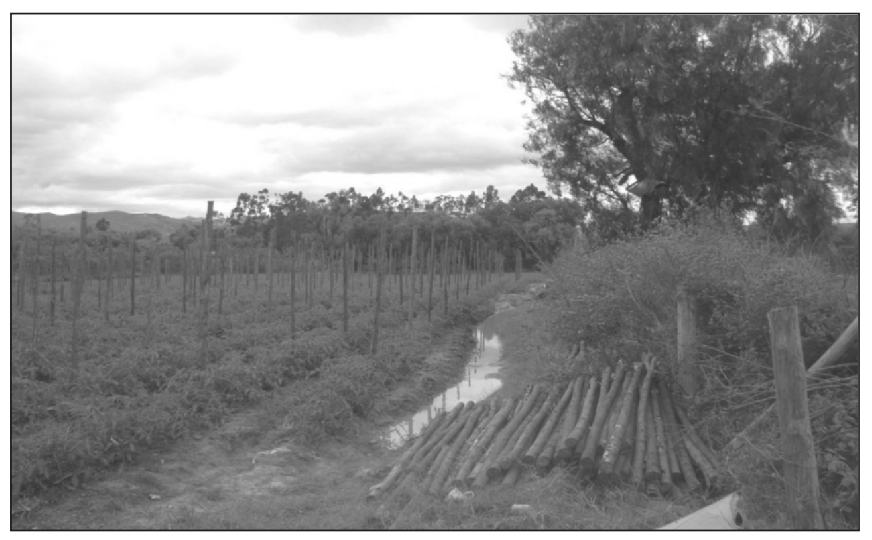

El aporte de agua a los cultivos implica tener disponibilidad de fuentes de agua no contaminada, libre de sedimentos y de metales pesados, para ser aplicada, de manera que 
esté libre de residuos orgánicos, estiércol humano y de animales y libre de agroquímicos. El uso de agua de acueductos veredales y municipales respecto a regulación de cantidades y frecuencia del riego no está registrado, en ninguno de los dos casos, de manera que se observa falta de monitoreo del volumen de agua a utilizar lo mismo que de su calidad ya que ello incide en la salud del hombre.

\section{BPA en asistencia técnica}

La verificación del riesgo considera que para establecer un cultivo en el que se tengan presentes las BPA deben proveerse de servicios de asistencia técnica con el fin de que cada una de las etapas del cultivo esté acompañada y asesorada debidamente. El asistente técnico debe visitar frecuentemente el predio recopilar información y registrar en el formato de valoración y evaluación de riesgos cualquier observación respecto a los procesos de planeación del cultivo.

\section{BPA en aplicación de plaguicidas}

La evaluación de riesgo en manejo fitosanitario permite identificar los peligros para la protección de la salud humana. Para el caso de tomate y de cebolla de bulbo se tiene en cuenta en un $10 \%$ en el primer cultivo y en un $5 \%$ en el segundo. No se cuenta con caracterizaciones del tipo nivel de exposición, por cuanto las investigaciones sobre el nivel de seguridad en el uso de plaguicidas, fertilizantes en los cultivos no se ha asumido, de manera que el desarrollo de investigaciones, el uso de modelos matemáticos, la consulta a expertos es exiguo. Se emplean alrededor de 30 aplicaciones en los dos cultivos de mezclas de fungicidas y de plaguicidas lo cual tiene consecuencias graves al destruir posibles insectos benéficos que pueden, en un momento determinado, controlar biológica y naturalmente dichas plagas y, hongos y bacterias que afectan a las plantas, alterando el equilibrio biológico existente. Los ingredientes activos más utilizados, insecticidas y fungicidas, son: Triflumuron $48 \%$ para control de polilla del tomate, Ciflutrina 5\%, Cipermetrinas, para pulgones, chinches y mosca blanca se usa el Metamidofos insecticida de amplio espectro y actividad sistémica, Metomilo de acción por contacto e ingestión en tomate larga vida, para nematodos el Fenamifos 10\%, y productos a base de Aldycar y Cipermetrina; aún se utilizan los productos organofosforados, Clorpirifos, producto no sistémico insecticida de contacto, productos a base de Diazinon que sirven para control de ácaros. En cebolla cabezona se utilizan productos para el control de enfermedades a base de Mancozeb.

No se cuenta con parámetros que permitan llevar un registro sobre límites máximos permisibles para el caso de insecticidas y de fungicidas recomendadas por el Codex Alimentarius para el caso de tomates y cebollas tratados con estos productos ni sobre los tiempos de espera para la recolección de los productos después de realizar las fumigaciones. La aplicación de insecticidas y fungicidas se hace desde que están las plántulas en almácigo, las dosis son mayores a lo recomendado en el $80 \%$ de los casos; en la cebolla cabezona generalmente se abandonan bulbos en el terreno recién cosechado lo que acarrea problemas fitosanitarios por proliferación de patógenos fúngicos especialmente, al cultivo de la cebolla 
no se le aplica el MIP o manejo Integrado de plagas y se convierte en monocultivo con las consecuentes infestaciones ciclo tras ciclo. Se requiere un plan de rotación de cultivos.

En el MIP sólo el 10\% de los cultivadores utilizan trampas y plantas con sustancias repelentes lo mismo que sustancias o extractos de plantas para control de hongos y de plagas. Se está utilizando por lo menos un $80 \%$ de productos de alta toxicidad que pueden generar problemas en la salud y cuyo uso está restringido por la Unión Europea. Los productos son generalmente seleccionados por el agricultor. No existe un documento escrito sobre la frecuencia de aplicación de un producto fitosanitario determinado, con el fin de llevar el record de aplicación y el impacto causado en el cultivo.

\section{BPA en condiciones de semillero}

El material de propagación a utilizar debe partir de una ficha técnica que incluye garantía respecto a la las condiciones de obtención de semilla, pruebas realizadas, producción esperada, porcentaje de germinación, para el caso de los dos cultivos objeto de estudio se conoce el origen de la semilla y se utiliza semilla de calidad. El personal que realiza las labores de propagación no se encuentra capacitado técnicamente en un $60 \%$, de manera que se observan deficiencias en la desinfección de los germinadores, humectación inadecuada de los sustratos, falta de homogeneidad en cuanto a profundidades de siembra.

Manejo ambiental: no se tiene en cuenta en el $80 \%$ de los casos con plan de manejo ambiental para el cultivo que contemple orientaciones respecto a la disposición de subproductos, manejo adecuado del agua, acciones tendientes a la protección de la vida de los recursos naturales, como el evitar la pérdida de la vida del suelo, y la necesidad de conservar y evitar la contaminación del agua. Es importante que cada una de las fincas considere transversalmente la calidad de vida, higiene y salud de los operarios y familias previniendo la toxicidad por el uso de productos químicos y biológicos.

\section{BPA en seguridad, bienestar y salud de los productores y sus familias}

La zona cuenta con Centros de Salud para atender casos de emergencia a trabajadores de las diferentes fincas, sin embargo, los trabajadores suelen afiliarse al SISBEN, en un 85\% aunque un $15 \%$ no tienen afiliación a este servicio. Para servicios de pensión sólo el $20 \%$ de la población trabajadora se encuentra afiliada.

\section{CONCLUSIONES}

La trazabilidad se debe llevar con la ayuda de registros y permite conocer cuáles son las condiciones de sanidad y de higiene del producto. Son aspectos a tener en cuenta en los mercados con el fin de obtener inocuidad y buenos precios. 
La planificación contable es otro aspecto que se considera en un $10 \%$ en los cultivos, el productor lleva sus cuentas en forma empírica pero no cruza la información de gastos y beneficios lo cual no le permite conocer y comprender contablemente su negocio

La biodiversidad se está viendo afectada en cuanto que a mayor establecimiento de sistemas de invernadero, se deforestan áreas importantes que sostienen el equilibrio eco sistémico, muchas plantas han sido arrasadas viéndose afectada la reserva de agua.

El manejo de agroquímicos sigue siendo irracional con contaminación de aguas y suelos. El impacto ambiental que se genera por el establecimiento de los sistemas de invernadero para el cultivo del tomate larga vida, ha generado destrucción de comunidades vegetales propias de la región como el hayuelo, el fique, la motua, la jarilla, el drago, el espino, los cactus algunos pajonales entre otras especies.

Respecto al recurso hídrico las fuentes de agua han disminuido su capacidad, y se adolece de un plan de manejo a nivel de finca para que el agricultor preserve ese recurso.

El $80 \%$ de los agricultores se encuentran asociados no ocurre lo mismo con los productores de cebolla que adolecen de sistemas de asociación que les apoye la producción, la financiación y la comercialización del producto. La organización de las comunidades es fundamental para generar sostenibilidad de los procesos inherentes a los sistemas de producción.

Se deben establecer capacitaciones a los productores sobre seguridad industrial, primeros auxilios, manejo del botiquín, prevención de riesgos, atención de emergencias No se cuenta con registros sobre entrenamiento de cada operario, tampoco se realizan controles de salud periódicamente sobre todo a las personas que realizan las aplicaciones químicas en los cultivos con el fin de verificar sus condiciones. El 70\% de los trabajadores participa de jornadas de salud realizadas por el Municipio y los puestos de salud.

\section{REFERENCIAS BIBLIOGRÁFICAS}

BOLETÍN INFORMATIVO. NÚM. 21. 2009, Implementación de las Normas sanitarias y el Sistema de Gestión de la Calidad como estrategia de los actores de la cadena de abastecimiento de alimentos. Bogotá.

DOCUMENTO CONPES 3375, Consejo Nacional de Política Económica y Social. Política Nacional de sanidad agropecuaria e inocuidad de alimentos para el sistema de medidas sanitarias y fitosanitarias.

GUILLÉN L. Percepción de los agricultores sobre el manejo integrado de plagas en el cultivo del tomate ( Lycopersicum esculentum). Revista ISSN 1690-07.

MINISTERIO DE AGRICULTURA y Desarrollo rural. Manual Buenas Prácticas Agrícolas. BPA. 
REVISTA SOCOLEN. Seminario manejo de plagas en frutales.

VÁZQUEZ, C y otros. (2004), Extracción e nutrientes por el cultivo del tomate (Lycopersicum esculentum) bajo riego por goteo en la Comarca de Lagunera. Agrofaz, ISSN 16658892. Vol. 4.

VARGAS, Abel. (2006), Estudio de comportamiento agronómico de variedades de tomate (Lycopersicum esculentum), producido orgánicamente. Universidad San Simón. 